\title{
A boundary element formulation for axi-symmetric problems in poro-elasticity
}

\author{
M. H. Ozyazicioglu ${ }^{1} \&$ M. Yener Ozkan ${ }^{2}$ \\ ${ }^{1}$ Ataturk University Turkey \\ ${ }^{2}$ Middle East Technical University, Turkey
}

\begin{abstract}
A formulation is proposed for the boundary element analysis of poro-elastic media with axi-symmetric geometry. The boundary integral equation is reduced to a set of line integral equations in the generating plane for each of the Fourier coefficients, through complex Fourier series expansion of boundary quantities in circumferential direction. The method is implemented into a computer program, where the fundamental solutions are integrated by Gaussian Quadrature along the generator, while Fast Fourier Transform algorithm is employed for integrations in circumferential direction. The strongly singular integrands in boundary element equations are regularized by a special technique. The Fourier transform solution is then inverted in to $\mathrm{R} \theta \mathrm{z}$ space via inverse FFT. The success of the method is assessed by problems with analytical solutions. A good fit is observed in each case, which indicates effectiveness and reliability of the present method.
\end{abstract}

Keywords: poro-elasticity, boundary element method, axi-symmetric, fast Fourier transform, wave propagation.

\section{Introduction}

Axi-symmetric boundary element formulations for elasto-dynamics $[1,2]$ and acoustics $[3,4]$ are available in the literature. However, these formulations are either fully axi-symmetric (both geometry and boundary conditions are axisymmetric) or they expand the boundary quantities into symmetric and antisymmetric modes, the final response is obtained by combining solutions for each of these modes. Accurate evaluation of either elliptic integrals or integrations in 
circumferential direction is required in the first and second approaches respectively, leading to extended run times.

Following the second approach described above, an axi-symmetric formulation for poro-elasticity is given by Dargush and Chopra [5]. Their formulation is fully axi-symmetric.

An alternative method based on complex Fourier series expansion of boundary quantities is developed by Özkan and Mengi [6]. In this method, the integrations in circumferential direction are accomplished by the Fast Fourier Transform (FFT) algorithm, which brings in considerable savings in computations.

The formulation of Tsepoura and Polyzos [7] for gradient elasticity is similar to [6]; however, they used the so called non-periodic FFT for integrations in $\theta$ direction when the source point lies in the integration element.

In this study, we extend the method in [6] to axi-symmetric poroelastodynamics with general boundary conditions.

The formulation has two major advantages: First, the use of FFT algorithm for integrations over $\theta$-direction increases computational performance considerably compared to Gaussian Quadrature for the same purpose. Second, using complex Fourier expansion obviates the analysis for symmetric and antisymmetric modes separately therefore, analysis for a general boundary condition is accomplished in a single run.

The formulation is developed in frequency domain, yet solutions in time domain can be obtained by inverse FFT, after solution vectors have been obtained for a sufficient number of frequencies.

\section{Biot theory of poro-elasticity}

Biot's poro-elasticity theory [8] is a generalization of elastodynamics to materials with fluid filled pore spaces and it includes Terzaghi's 1-D consolidation theory as a special case. This theory led to reformulation of problems in soil mechanics, geophysics, acoustics and biomechanics [9] predicting behaviour beyond that conceivable by classical elasticity theory. The predictions of the theory have been substantially verified experimentally [10, 11]. The material constants involved are easily discernible, physically meaningful and experimentally measurable. The theory also brings in a stronger definition of effective stress, which is a fundamental concept in soil mechanics.

\subsection{Governing equations in frequency domain}

Governing equations of poro-elasticity [11] in frequency domain are given as

$$
\begin{gathered}
\mu u_{i, j j}+(\lambda+\mu) u_{j, j i}-(\alpha+\beta) p_{, i}+f_{i}=-\omega^{2}\left(\rho+\beta \rho_{f}\right) u_{i} \\
-\beta \frac{1}{\omega^{2} \rho_{f}} p_{, k k}+(\alpha+\beta) u_{k, k}+\frac{1}{Q} p=\frac{1}{i \omega} a
\end{gathered}
$$


where, $u_{i}, p$ are Fourier transforms of displacement components and porepressure, $f_{i}, a$ are body force and fluid generation per unit volume in frequency domain respectively. The Fourier and inverse Fourier transform of a time dependant function $F(t)$ is defined by the following pair

$$
f(\omega)=\int_{-\infty}^{\infty} F(t) e^{-i \omega t} d t \leftrightarrow \mathrm{F}(\mathrm{t})=\frac{1}{2 \pi} \int_{-\infty}^{\infty} f(\omega) e^{i \omega t} d \omega
$$

Other parameters in (1) and (2) are

$\omega$ the circular frequency.

$\left.\begin{array}{l}\mu \\ \lambda \\ \alpha \\ Q\end{array}\right\}$

$\left.\begin{array}{l}\rho \\ \rho_{f}\end{array}\right\}$ unit mass for bulk material and interstitial fluid

and $\beta$ is defined as

$$
\beta=\frac{n^{2} \kappa \rho_{f} \omega^{2}}{i \omega n^{2}-\omega^{2} \kappa\left(\rho_{a}+n \rho_{f}\right)}
$$

where, porosity and permeability are designated respectively by $\mathrm{n}$ and $\kappa$. Finally, $\rho_{a}$ is the added mass density.

\subsection{The boundary integral equation (BIE)}

The weighted residual statement of (1) and (2) is obtained by taking the inner product of the system with a vector of weighting functions $\left[\begin{array}{ll}u_{i}^{*} & p^{*}\end{array}\right]^{T}$

$$
\int_{\Omega}\left[\begin{array}{c}
u_{i}^{*} \\
p^{*}
\end{array}\right]^{T}\left([\mathbf{B}]\left[\begin{array}{c}
u_{i} \\
p
\end{array}\right]+\{F\}\right) d \Omega=0
$$

where, $\{\mathrm{F}\}=\left[\begin{array}{c}\mathrm{f}_{\mathrm{i}} \\ \mathrm{a}\end{array}\right]$, and $[\mathrm{B}]$ represent the partial differential operator pertaining to equations (1) and (2). Using Gauss Integral Theorem, in the absence of body forces and fluid source (4) can be converted to the following BIE

$$
\underline{c} \underline{u}(A)=\int_{\Gamma} \underline{G^{\prime}}(A, P) \underline{t}(P) d \Gamma-\int_{\Gamma} \underline{H^{\prime}}(A, P) \underline{u}(P) d \Gamma
$$

where, an underline designates a matrix; $\underline{G}^{\prime}$ and $\underline{H^{\prime}}$ matrices $(4 \times 4)$ contain first and second fundamental solutions of poro-elastodynamics, respectively. These 
solutions are two point (source at A, response at P) functions and associated with an infinite medium with either a point force in only one coordinate direction in turn or a unit fluid injection rate at a point "A". The point "P" is called the integration point. $\underline{\mathrm{t}}$ and $\underline{\mathrm{u}}$ are $(4 \times 1)$ column matrices representing generalized traction and displacement vectors at the boundary points, $\underline{\mathrm{c}}$ is a $(4 \times 4)$ matrix, which is $c_{i j}=0.5 \delta_{i j}$ on a smooth boundary.

\section{Boundary element formulation for axi-symmetric bodies}

Consider a poro-elastic axi-symmetric body of boundary $\mathrm{S}$, referred to a cylindrical coordinate system $\mathrm{R}-\theta-\mathrm{z}$ as shown in fig. 1 , where $\mathrm{z}$ is the axis of revolution. It will be assumed that the boundary conditions are not axisymmetric. The method is based on complex Fourier series expansion of the boundary quantities (displacements, pore-pressure, tractions and normal component of fluid flux vector) in circumferencal direction.

There are two main advantages of this method [6] over others available in the literature [1-5]:

i) the evaluation of integrals in $\theta$ direction is accomplished by FFT algorithm, which reduces the computational load,

ii) the need for differentiating symmetric and anti-symmetric modes in the analysis is eliminated, which facilitates computer programming.

The transformation from cylindrical to cartesian coordinates is given by:

$$
\underline{t}=\underline{Q} \underline{t}_{c} \quad \text { and } \quad \underline{u}=\underline{Q} \underline{u}_{c}
$$

where, where, $\underline{u_{c}}$ and $\underline{t_{c}}$ are the generalized displacement and traction vectors cylindrical coordinate frame. The transformation (rotation) matrix $\underline{Q}$ is

$$
\underline{Q}=\left[\begin{array}{cccc}
\cos \alpha & -\sin \alpha & 0 & 0 \\
\sin \alpha & \cos \alpha & 0 & 0 \\
0 & 0 & 1 & 0 \\
0 & 0 & 0 & 1
\end{array}\right]
$$

Substituting (6) in (5) and multiplying from left by $\mathrm{Q}^{\mathrm{T}}(\mathrm{A})$, one gets

$$
\underline{c}_{\mathrm{c}} \underline{u}_{c}(A)=\int_{C}^{2 \pi} \int_{0}^{2} \underline{G}_{c}(A, P) \underline{t}_{c}(P) R d \theta d s-\int_{C}^{2 \pi} \int_{0} \underline{H}_{c}(A, P) \underline{u}_{c}(P) R d \theta d s
$$

Since, $d \Gamma=R d \theta d s$. (8) is now the BIE in cylindrical coordinates where $\underline{\mathrm{G}}_{\mathrm{c}}(\mathrm{A}, \mathrm{P})$ and $\underline{\mathrm{H}}_{\mathrm{c}}(\mathrm{A}, \mathrm{P})$ represent the fundamental solution matrices

$$
\begin{aligned}
& \underline{G}_{c}(A, P)=\underline{Q}^{T}(A) \underline{G^{\prime}}(A, P) \underline{Q}(P) \\
& \underline{H}_{c}(A, P)=\underline{Q}^{T}(A) \underline{H^{\prime}}(A, P) \underline{Q}(P)
\end{aligned}
$$

and $\underline{c_{c}}=\underline{c}$ is the free term coefficient. 
It can be shown that the fundamental solutions $\underline{G}_{c}(A, P)$ and $\underline{H}_{c}(A, P)$ are functions of the form

$$
\underline{\mathrm{G}}_{\mathrm{c}}\left(\mathrm{r}, \theta-\theta^{\prime}, \mathrm{z}-\mathrm{z}^{\prime}\right), \underline{\mathrm{H}}_{\mathrm{c}}\left(\mathrm{r}, \theta-\theta^{\prime}, \mathrm{z}-\mathrm{z}^{\prime}\right)
$$

where, primed variables represent source point coordinates.

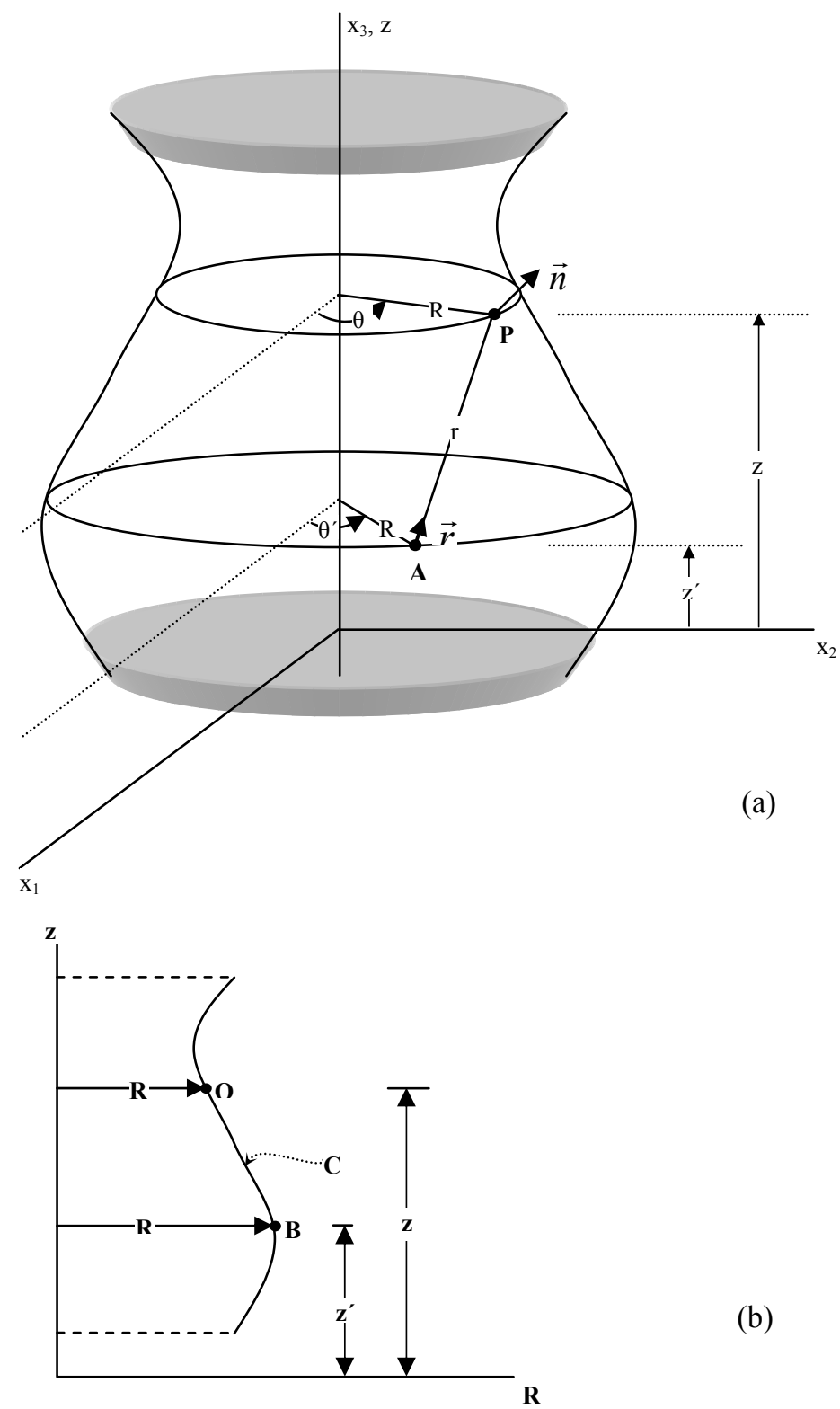

Figure 1: An axi-symmetric body referred to $\mathrm{R} \theta \mathrm{z}$ coordinate system: a) three dimensional body, b) x-section on R-z plane. 


\subsection{Expansion of field variables in complex Fourier series}

It follows from axi-symmetric geometry that the boundary quantities are " $2 \pi$ " periodic in angular direction, thus can be expand into complex Fourier series:

$$
\begin{aligned}
& \underline{u}_{c}(R, \theta, z)=\sum_{k=-\infty}^{\infty} \underline{u}_{c}^{k}(R, z) e^{i k \theta} \\
& \underline{t}_{c}(R, \theta, z)=\sum_{k=-\infty}^{\infty} \underline{t}_{c}^{k}(R, z) e^{i k \theta}
\end{aligned}
$$

when (11) is substituted, (5) is reduced, for a k-th term of the Fourier series,

$$
\begin{aligned}
\underline{c}_{c} \underline{\tilde{u}}_{c}^{k}\left(R^{\prime}, z^{\prime}\right)=\int_{C} \underline{G}_{c}^{k}\left(R^{\prime}, z^{\prime} ; \mathrm{R}, \mathrm{z}\right) \underline{\tilde{t}}_{c}^{k}(R, z) R d s- \\
\\
\quad \int_{C} \underline{H}_{c}^{k}\left(R^{\prime}, z^{\prime} ; \mathrm{R}, \mathrm{z}\right) \underline{\tilde{u}}_{c}^{k}(R, z) R d s
\end{aligned}
$$

where the integrals are along the generating curve, thus, the dimensionality is reduced by one, at the expense of now $\mathrm{k}$ boundary equations. The equations are exact with infinite terms, but a good approximation is generally obtained by truncating the series to only a few terms. After Fourier coefficients are computed from (12) for each $\mathrm{k}$, boundary quantities in $\mathrm{R} \theta \mathrm{z}$ space can be evaluated by inverse FFT. When spatial discretization with constant elements along the generating curve is introduced (12) becomes

$$
\underline{c}_{c} \underline{\tilde{u}}_{c i}^{k}=\sum_{j} \underline{\widetilde{G}}_{i j}^{k} \underline{\tilde{t}}_{c j}^{k}-\sum_{j} \underline{\widetilde{H}}_{i j}^{k} \underline{\tilde{u}}_{c j}^{k}
$$

where, $\underline{\widetilde{u}}_{c j}^{k}$ and $\underline{\underline{t}}_{c j}^{k}$ are the values of $\underline{\tilde{u}}_{c}^{k}$ and $\underline{\underline{t}}_{c}^{k}$ over the element “ $j$ ”, and

where

$$
\begin{gathered}
\widetilde{G}_{i j}^{k}=\int_{0}^{2 \pi} \underline{\mathrm{G}}_{i j}(\theta) e^{i k \theta} d \theta \\
\underline{\widetilde{H}}_{i j}^{k}=\int_{0}^{2 \pi} \underline{\mathrm{H}}_{i j}(\theta) e^{i k \theta} d \theta \\
\underline{\mathrm{G}}_{\mathrm{ij}}(\theta)=\left.\int_{\mathrm{C}_{\mathrm{j}}} \underline{\mathrm{G}}_{\mathrm{c}}(\underbrace{\mathrm{R}^{\prime}, \mathrm{z}^{\prime}}_{\mathrm{Q}_{\mathrm{i}}} ; \underbrace{\mathrm{R}, \mathrm{z} ; \theta-\theta^{\prime}}_{\mathrm{Q}})\right|_{\theta^{\prime}=0} \mathrm{R} \mathrm{ds} \\
\underline{\mathrm{H}}_{\mathrm{ij}}(\theta)=\left.\int_{\mathrm{C}_{\mathrm{j}}} \underline{\mathrm{H}}_{\mathrm{c}}(\underbrace{\mathrm{R}^{\prime}, \mathrm{z}^{\prime}}_{\mathrm{Q}_{\mathrm{i}}} ; \underbrace{\mathrm{R}, \mathrm{z} ; \theta-\theta^{\prime}}_{\mathrm{Q}})\right|_{\theta^{\prime}=0} \mathrm{R} \mathrm{ds}
\end{gathered}
$$

In system form, (13) may be written as

$$
\underline{\widetilde{H}}^{k} \underline{\tilde{u}}^{k}=\underline{\widetilde{G}}^{k} \underline{\tilde{t}}^{k}
$$

where, 


$$
\begin{aligned}
& \underline{\widetilde{G}}^{k}=\left\lfloor\widetilde{G}_{i j}^{k}\right\rfloor \\
& \underline{\widetilde{H}}^{k}=\left[\underline{\widetilde{H}}_{i j}^{k}+\frac{1}{2} \underline{I}_{i j}\right]
\end{aligned}
$$

Fast and accurate evaluation of the integrals in (14) can be accomplished by FFT. This is the third stage we use FFT algorithm for computations. It should be noted that different number of Fourier terms may be selected for Complex Fourier expansion in (11) and in the integral evaluations in (14).

The computational procedure can be summarised as follows:

- Choose $\mathrm{N}^{\prime}=2^{\mathrm{Mp}}$, the number of terms in Complex Fourier Series, and compute Fourier coefficients of boundary excitations by FFT $\left(4^{\text {th }}\right.$ FFT).

- Choose $\mathrm{N}=2^{\mathrm{M}}$, the number of division along $\theta$ for integrals in (14)

- Discretize the generator and let the number of boundary elements be $\mathrm{m}$.

- Compute $\underline{G}_{i j}^{s}$ and $\underline{\tilde{H}}_{i j}^{s}(\mathrm{~s}=0 \ldots \mathrm{N}-1)$, and form the system matrices $\underline{\tilde{H}}^{k}$ and $\underline{G}^{k}$ for $k=0 \ldots \mathrm{N}^{\prime}-1$. A frequency shift is necessary when assembling $\underline{\widetilde{G}}_{i j}^{s}$ and $\underline{\tilde{H}}_{i j}^{s}$ into $\underline{\widetilde{G}}^{k}$ and $\underline{\tilde{H}}^{k}$, as "s" and "k" run through different ranges. The singular integrals when source point is on the integration element is circumvented by introducing a panel element around the generator, details can be found in [11].

- Solution of the complex algebraic system of equations (15) together with the specified boundary conditions yields the Fourier coefficients $\underline{u}^{k}$ and $\tilde{t}^{k}$ at frequency points $k=0,1,2, \ldots, \mathrm{N}^{\prime}-1$.

- By an inverse FFT $\left(5^{\text {th }}\right)$ evaluate the boundary quantities in $(R, \theta, z)$ space.

- The BIE (5) with $\underline{\mathrm{c}}=\underline{\mathrm{I}}$ is used to compute solution at an internal point if required.

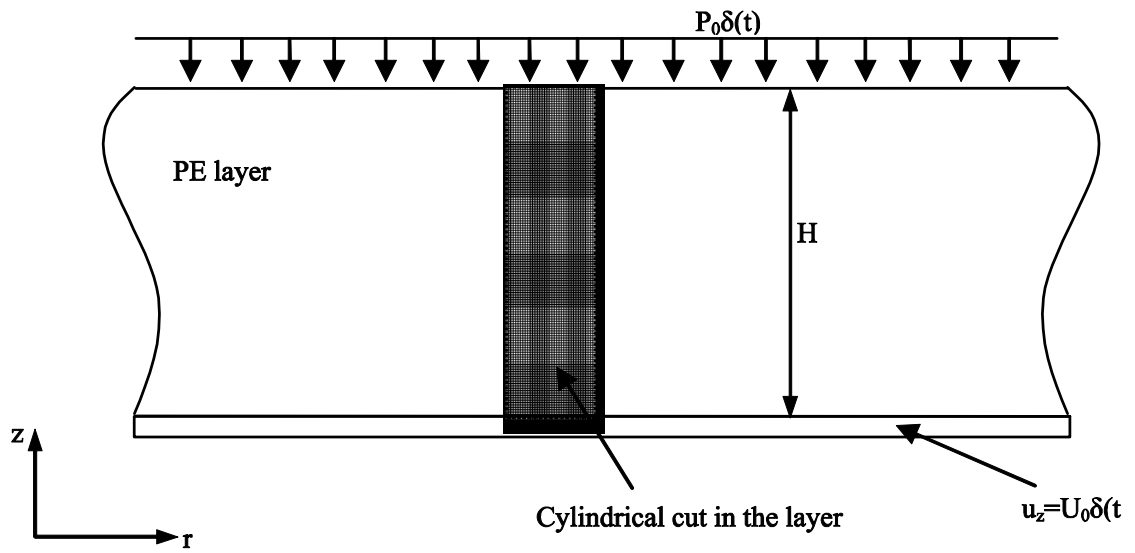

Figure 2: One dimensional wave propagation in a layer. 


\section{Assessment of the formulation}

A computer program called AxiPoro is implemented using above algorithm with standard $\mathrm{C}$ language. And the results are assessed against three problems which have exact analytical solutions.

\subsection{One dimensional wave propagation in a PE layer}

This problem, in the context of poro-elasticity is first mentioned in [12], an analytical solution is also provided in the same reference.

In solving this problem by BEM, we model the PE "layer" by a PE "column" of unit diameter as shown in fig. 3. Although, the PE column is not an exact model for the layer, still is a close approximation. The material properties are given in Table 1 below.
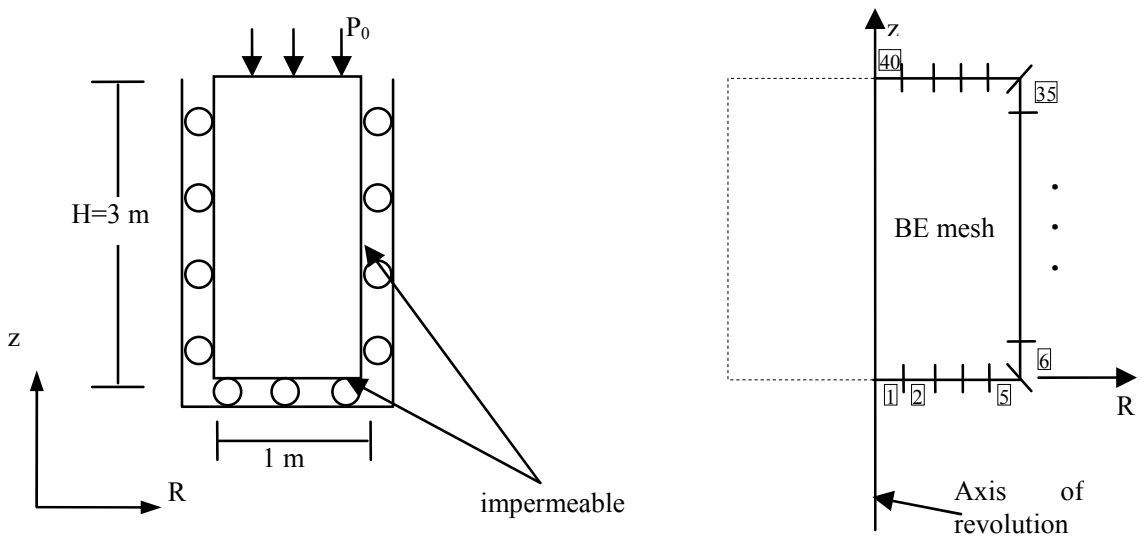

Figure 3: $\quad$ Equivalent column model of PE layer for BE analysis.

Table 1: $\quad$ Material data for Berea sand stone.

\begin{tabular}{|c|c|c|c|c|c|c|c|c|}
\hline $\mathrm{N}$ & $\alpha$ & $\mathrm{Q}(\mathrm{Pa})$ & $\mu(\mathrm{Pa})$ & $v$ & $\begin{array}{c}\kappa \\
\left(\mathrm{m}^{4} / \mathrm{N} / \mathrm{s}\right)\end{array}$ & $\begin{array}{c}\rho \\
\left(\mathrm{kg} / \mathrm{m}^{3}\right)\end{array}$ & $\begin{array}{c}\rho_{\mathrm{f}} \\
\left(\mathrm{kg} / \mathrm{m}^{3}\right)\end{array}$ & $\begin{array}{c}\rho_{\mathrm{a}} \\
\left(\mathrm{kg} / \mathrm{m}^{3}\right)\end{array}$ \\
\hline 0.19 & 0.778 & $1.353^{*} 10^{10}$ & $6^{*} 10^{9}$ & 0.2 & $1.9 * 10^{-10}$ & 2458 & 1000 & 125.4 \\
\hline
\end{tabular}

The poro-elastic column problem is solved by program AxiPoro. The column is modelled by 40 axi-symmetric boundary elements. $\mathrm{N}=128\left(2^{7}\right)$ and $\mathrm{N}=32\left(2^{5}\right)$ sub-divisions for circumferential integrations were used for convergence checking. A slight hysteretic damping of $0.3 \%$ is also introduced. The results for top displacement are plotted in fig. 4 together with the analytical solution in [12]. 


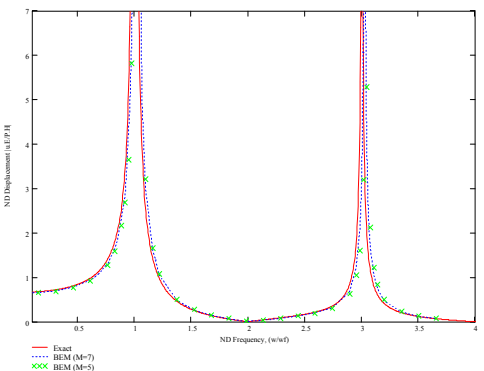

(a) Top displacement amplitude.



(b) Real part of top displacement.

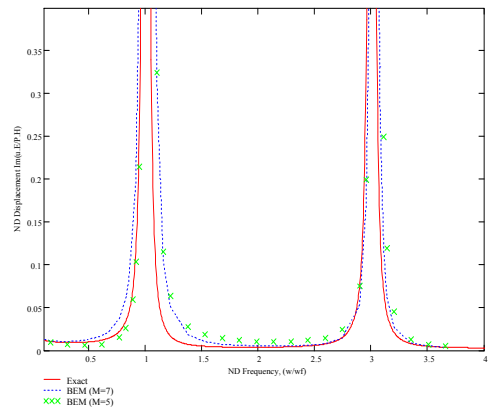

(c) Imaginary part of top displacement, $E=2 \lambda+\mu$.

Figure 4: $\quad$ PE column (traction B.C. at top): BEM vs. analytical solution.

\subsection{Sudden pressurization of a circular cavity}

This problem was investigated by Senjuntichai and Rajapakse [13].

However, the governing equations used in [13] did not include the "continuity equation", therefore, to comply with our BEM formulation, we re-work the solution for sudden pressurization (Dirac loading in time) with permeable wall condition; the analytical solution can be found in [11].

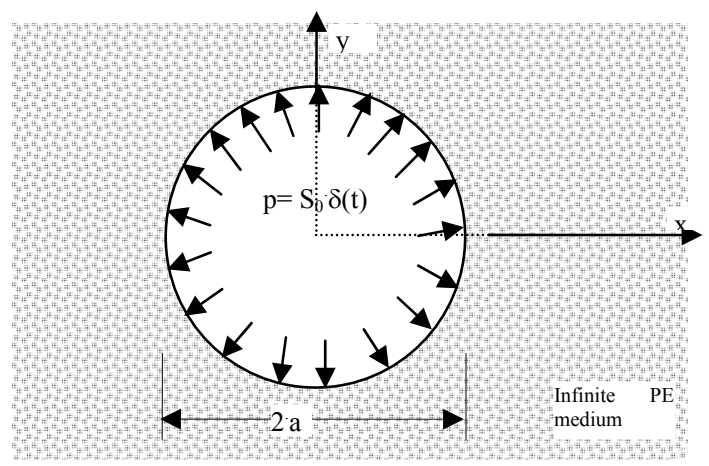

Figure 5: Circular cavity (infinite cylinder) in a poro-elastic full space suddenly pressurized. 
The poro-elastic circular cavity problem is solved by program AxiPoro, with the material data of Berea sandstone (Table 1). A finite cylindrical cavity of 10 metres height is modelled by 25 axi-symmetric boundary elements. The angular divisions for circumferential integrations are $\mathrm{N}=2^{7}=128$. A hysteretic damping of $5 \%$ is used to eliminate edge reflections as much as possible. The results for radial surface displacements (compliance) at mid-height of the cavity are plotted in fig. 6 together with the analytical solution. Slight noise is observed in the figures due to waves generated at the ends of the cavity.

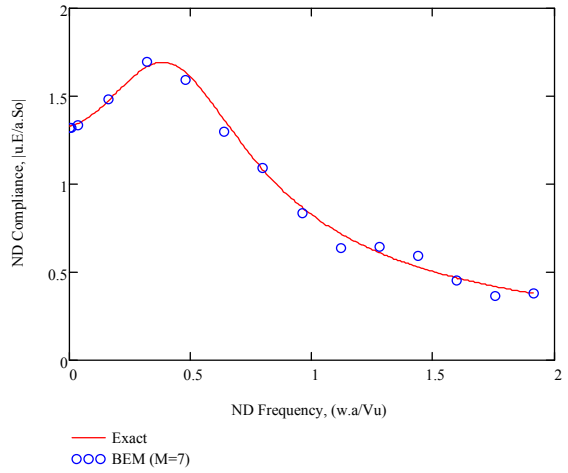

a) Absolute value of ND compliance.

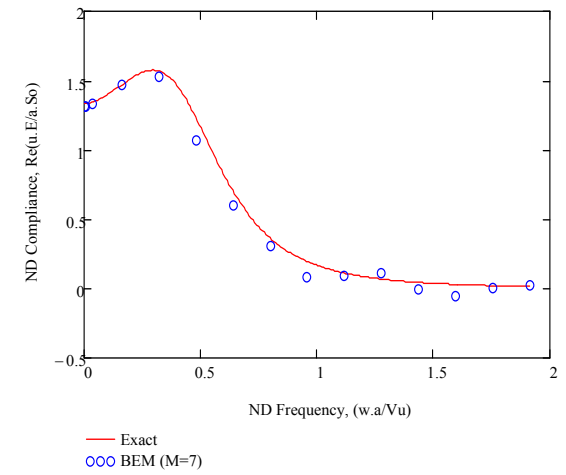

b) Real part of ND compliance.

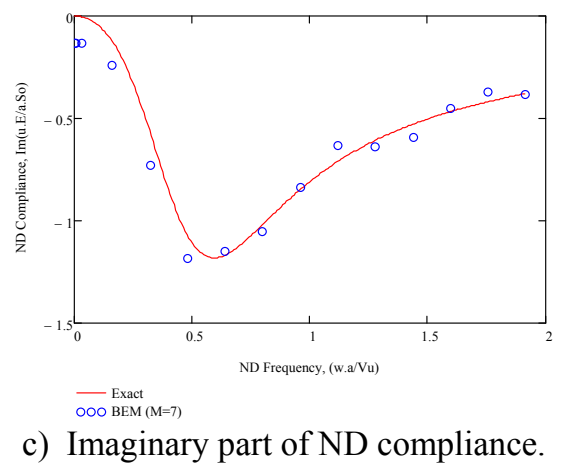

Figure 6: Circular cavity: $\mathrm{BEM}$ vs. analytic solution, $\mathrm{E}=2 \lambda+\mu$.

\section{Conclusions}

In this study, an axi-symmetric BE formulation is presented for dynamic poroelasticity. The method makes frequent uses of FFT as an effective computational tool. The proposed formulation has several advantages over others in the literature; to summarize

- expansion of boundary variables in complex Fourier series, obviates the need for differentiating symmetric and anti-symmetric modes in the 
analysis leading to easier coding in the case of arbitrary boundary conditions.

- use of FFT algorithm increases the computational performance and accuracy remarkably.

- convergence of the method for a given BE mesh is controlled by the number of subdivisions in circumferential direction, good accuracy is obtained for $\mathrm{N}=2^{8}$.

If response in time domain is required, the analysis can be repeated for sufficient number of frequencies $\omega$, and time history of boundary quantities can be evaluated by inverse FFT, again.

The computer implementation of the method is assessed against analytical solutions in the literature, a good fit is observed.

\section{References}

[1] Brebbia, C. A. and Dominguez, J., Boundary Elements An Introductory Course, Second Edition, Computational Mechanics Publications, UK, 1992.

[2] Becker, A. A., The Boundary Element Method in Engineering, McGraw Hill, London, 1992.

[3] Juhl, P., An axisymmetric integral equation formulation for free space non-axisymmetric radiation and scattering of a known incident wave, J. Sound and Vibration, V. 163, pp. 397-406, 1993.

[4] Pozrikidis, C., A Practical Guide to Boundary Element Methods with the Software Library BEMLIB, CRC Press, May 15 2002, New York, 2002.

[5] Dargush, G. F. and Chopra, M. B., Dynamic analysis of axisymmetric foundations on poroelastic media, J. Eng. Mech., ASCE, V.122, No.7, pp. 623-632, 1996.

[6] Özkan, G., Mengi, Y., On the use of FFT algorithm for the circumferential coordinate in boundary element formulation of axisymmetric problems, 40(13), pp.2385-2412, 1997.

[7] Tsepoura, K. G. and Polyzos, D., Static and harmonic BEM solutions of gradient elasticity problems with axisymmetry, Computational Mechanics, V.32, pp.89-103, 2003.

[8] Biot, M. A., The theory of propagation of elastic waves in a fluid saturated porous solid, I. Low frequency range, J Acoust. Soc. Am., V.28, pp. 168-178, 1956.

[9] Cowin, S. C., Bone poroelasticity, J. Biomech., V.32, pp.217-238, 1999.

[10] Plona, T. J., Observation of a second bulk comp. wave in a porous medium at ultrasonic frequencies, Appl. Phys. Lett., V.36, pp. 259-261, 1980.

[11] Ozyazicioglu, M., A boundary element formulation for axi-symmetric poroelasticity, Ph. D. Thesis, Middle East Technical University, Dept. of Civ. Eng., Ankara, Turkey, 2006.

[12] Cheng, A. H-D.; Badmus, T.; Beskos, D. E., Integral equation for dynamic poroelasticity in frequency domain with BEM solution, J. Eng. Mech., ASCE, Vol. 117, No. 5, pp. 1136-1157, 1991. 
176 Boundary Elements and Other Mesh Reduction Methods XXXIII

[13] Senjuntichai, T. and Rajapakse, R. K. N. D., Transient response of a circular cavity in a poroelastic medium, Int. J. Num. Analyt. Meth. Geomech., V.17, pp.357-383, 1993. 